\title{
System Coverage and Capacity Analysis on Millimeter-Wave Band for 5G Mobile Communication Systems with Massive Antenna Structure
}

\author{
Jun Suk Kim,, Jae Sheung Shin, ${ }^{2}$ Sung-Min Oh, ${ }^{2}$ Ae-Soon Park, ${ }^{2}$ and Min Young Chung ${ }^{1}$ \\ ${ }^{1}$ College of Information and Communication Engineering, Sungkyunkwan University, 2066 Seobu-Ro, Jangan-Gu, Suwon, \\ Gyeonggi-Do 440-746, Republic of Korea \\ ${ }^{2}$ Mobile Access Research Division, Electronics and Telecommunications Research Institute, 138 Gajeongno, Yuseong-gu, \\ Daejeon 305-700, Republic of Korea
}

Correspondence should be addressed to Min Young Chung; mychung@skku.edu

Received 21 February 2014; Revised 10 May 2014; Accepted 5 June 2014; Published 2 July 2014

Academic Editor: Ahmed Iyanda Sulyman

Copyright (C) 2014 Jun Suk Kim et al. This is an open access article distributed under the Creative Commons Attribution License, which permits unrestricted use, distribution, and reproduction in any medium, provided the original work is properly cited.

\begin{abstract}
The use of a millimeter-wave band defined as a $30-300 \mathrm{GHz}$ range is significant element for improving performance of 5th generation $(5 \mathrm{G})$ mobile communication systems. However, since the millimeter-wave signal has peculiar propagation characteristics especially toward non-line-of-sight regions, the system architecture and antenna structure for 5G mobile communications should be designed to overcome these propagation limitations. For realization of the 5G mobile communications, electronics and telecommunications research institute (ETRI) is developing central network applying various massive antenna structures with beamforming. In this paper, we have introduced the central network and evaluated the system coverage and capacity through $\mathrm{C}++$ language-based simulations with real geospatial information.
\end{abstract}

\section{Introduction}

As use of various multimedia services with smart devices has become more common, data traffic has rapidly increased in mobile communication networks [1]. Qualcomm, especially, expects that the volume of mobile traffic in 2020 will increase 1000 times more than that of the current traffic [2]. To accommodate the data traffic, current fourth generation (4G) mobile communication systems including long term evolution-advanced (LTE-A) and worldwide interoperability for microwave access networks (WiMAX) consider various advanced technologies such as multiple input and multiple output (MIMO), multicarrier transmission, and carrier aggregation $[3,4]$. In spite of these technologies, frequency bands below $3 \mathrm{GHz}$ used in the current mobile communication systems are expected to be crowded due to the increasing demand in mobile data traffic. Therefore, in order to enlarge the frequency band to accommodate the emerging data traffic, a new type of frequency band should be considered in 5th generation (5G) mobile communication systems.
The millimeter-wave band in the $30-300 \mathrm{GHz}$ range is being considered as a candidate for new radio bands for $5 \mathrm{G}$ mobile communication systems $[5,6]$. Since most of the millimeter-wave band is underutilized, 5G mobile communication systems are expected to utilize wide and continuous bands for radio access networks (RANs). By using the vast amounts of radio resources in the millimeterwave band, system capacity of the $5 \mathrm{G}$ mobile communication systems can be improved and mobile devices served by the system can experience better service environments with high-speed transmission and low latency compared to those of the current mobile communication systems. Therefore, a mobile communication system using the millimeter-wave band is expected to provide novel multimedia services with exacting service requirements.

In order to use the millimeter-wave band more efficiently, various advanced technologies are considered for use in 5G mobile communication networks. For example, beamforming technology can be considered to improve the spectral efficiency of mobile communication networks [7, 
8]. The beamforming technology facilitates spatial reuse of limited radio resources in mobile communication networks. A massive antenna structure (MAS) for forming multiple beams can also support the spatial reuse of a base station (BS) [9]. In addition, relay transmission is utilized to broaden system coverage and improve channel quality in the $5 \mathrm{G}$ mobile communication systems $[10,11]$. The relay transmission reduces the coverage hole where radio signals are hardly propagated. In particular, since the millimeter wave signals have poor propagation characteristics toward non-line-ofsight (NLoS) regions [12], multiple relay base stations (RBSs) should be deployed at proper geographical positions enabling to overcome the coverage holes [13].

Several researches and projects for the $5 \mathrm{G}$ mobile communications have been conducted in recent years $[14,15]$. At the University of Texas, the characteristics of the millimeterwave band at $38 \mathrm{GHz}$ for outdoor cellular communication channels have been evaluated based on experimentally measured received signal strength (RSS) in a real campus environment [14]. In the Dallas Technology Laboratory of Samsung Electronics, an adaptive antenna array structure is proposed for beamforming in the millimeter-wave band [15]. In addition, new radio frame structure and network architecture are introduced for a mobile broadband system operating in the millimeter-wave band.

For successful realization of the $5 \mathrm{G}$ mobile communications, it is important not only to design overall system concept and structure but also to evaluate and verify the system on system level. Therefore, this paper firstly introduces a novel system architecture for the $5 \mathrm{G}$ mobile communications, named central network, and explains related technologies in detail. And then, we represent system coverage and capacity of the central network through the simulation on considering real geospatial information. The remainder of the paper is organized as follows. Details of the central network are introduced in Section 2. Section 3 contains a systematic performance evaluation in terms of system coverage and capacity. Finally, Section 4 summarizes the paper and provides directions and prospects for future research.

\section{Central Network for 5G Mobile Communications}

In order to accommodate rapidly increasing mobile data traffic, mobile network operators (MNOs) have upgraded their $4 \mathrm{G}$ mobile networks by using small cell network architecture and advanced transmission technologies. However, despite the constant endeavor, the increasing mobile data traffic is expected to exceed the capacity of current $4 \mathrm{G}$ mobile networks and to cause severe traffic flood. Therefore many countries have launched different research and development projects for $5 \mathrm{G}$ mobile communication enabling to overcome capacious limitation of current $4 \mathrm{G}$ mobile networks. European union (EU) has made a massive investment in research project of $5 \mathrm{G}$ mobile communications, named as mobile and wireless communications enablers for the twentytwenty information society (METIS), and 5G public-private partnership (5GPPP) association is organized in order to

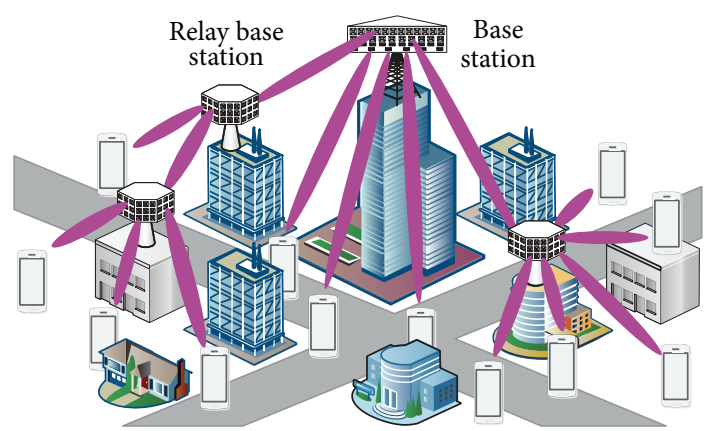

FIGURE 1: Radio access network architecture of the central network.

take the lead in technology/standard of 5G mobile communications [16, 17]. Moreover, China government has formed "international mobile telecommunication in the year 2020" (IMT-2020) project group, and Japan government has also established a new 5G working group called "2020 and Beyond Ad-Hoc" [18].

In Republic of Korea, electronics and telecommunications research institute (ETRI) is researching and developing $5 \mathrm{G}$ mobile communication system called central network. The central network is targeted to provide an ultra-high system capacity in about hundred Gbps in order to support various multimedia services and to accommodate huge amounts of mobile traffic generated by the services. To meet the requirement, the central network is considered to utilize $1 \mathrm{GHz}$ bandwidth in $27 \mathrm{GHz}$ band. For more effective utilization of the bandwidth, effective spatial division multiple access (SDMA) is considered based on massive antenna structure. Since the millimeter-wave band has a very short wavelength, the size of antennas can be miniaturized. This means that BS and mobile device can embed more antennas into their package.

Due to the poor propagation characteristics of the millimeter-wave band, it is difficult to secure good channel condition and sufficient system coverage in the central network. Thus, in order to overcome the poor propagation, narrow-width beamforming (pencil beamforming) is considered in the central network. Especially, the narrow width beam can maximize the gain from the use of SDMA. Moreover, the central network expands system coverage by using relay transmissions. As RBSs are installed with multilevel topology, the central network can reduce coverage holes and provide line of sight (LoS) connectivity to larger number of mobile devices. Figure 1 shows the overall system architecture of the central network with beamforming and the multilevel relay technology.

\subsection{Core Technologies for Central Networks}

2.1.1. Beamforming. Beamforming technology is a useful solution for overcoming the poor propagation of the millimeter-wave band and improving channel quality. Beamforming enables a transmitter to radiate a signal in a particular direction and allows a receiver to receive signals from a particular direction. This means that the wireless signal can 


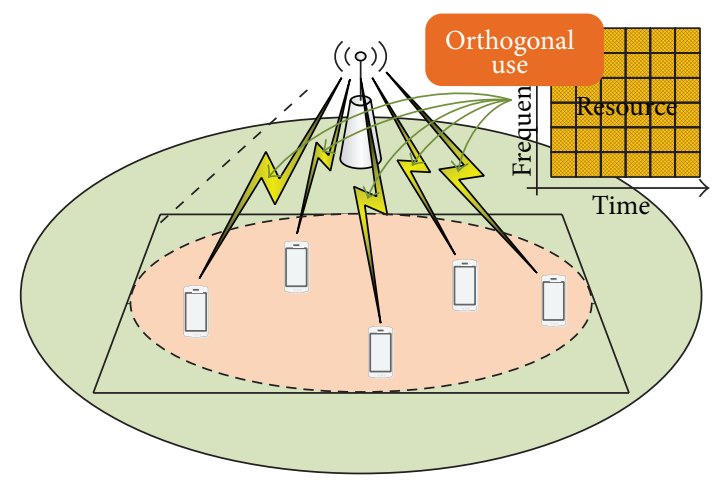

(a) Non-SDMA case

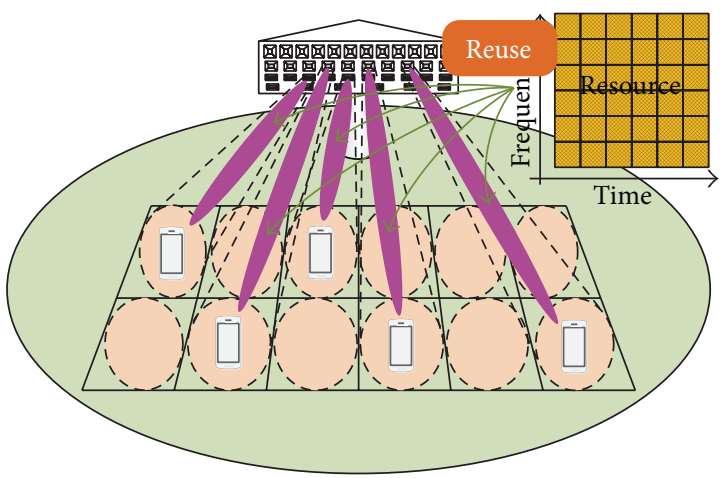

(b) SDMA case

FIGURE 2: Spatial division multiple access by using beamforming and massive antenna structure.

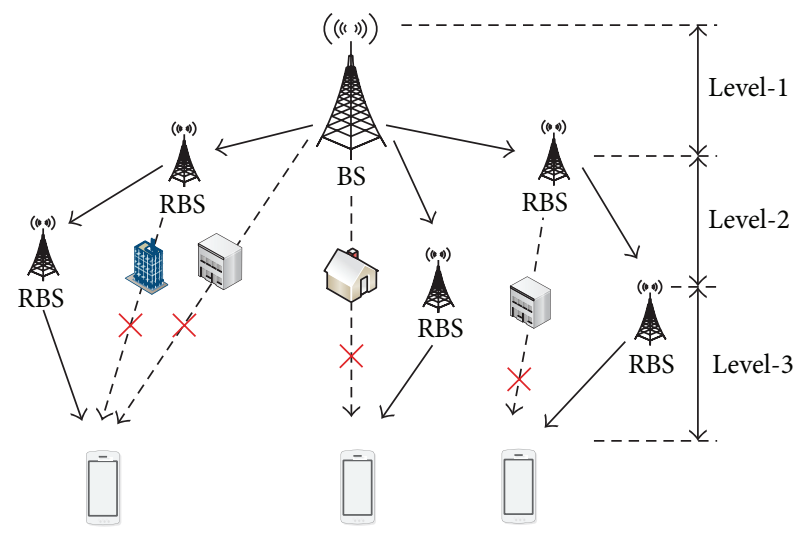

Figure 3: Multilevel topology through wireless backhaul link.

be better delivered in the particular direction and quality of the received signal can be improved. In addition, the beamforming makes it easy to distinguish desired signal and interference. As a result, the beamforming can be used to provide spatial division multiple access (SDMA) which is able to offer superior performance in radio multiple access communication systems. The strong straightforwardness of the millimeter-wave band helps to prevent a signal spread and interference. Therefore, the spectral efficiency of the central network can increase through spatial reuse with the prevention of signal spread and interference.

In general, two types of beamforming can be considered: fixed beamforming (switched beamforming) and adaptive beamforming. In fixed beamforming, a static set of beams is formed in each direction without consideration of the surrounding environment. On the other hand, in adaptive beamforming, the directions of the radiated beams are automatically adapted based on the surrounding environments. Even though adaptive beamforming enables more effective beamforming, but it requires more complex and redundant feedback operations than the fixed beamforming. Thus, we focus on switched beamforming in the central network. For cost-effective beamforming operation, we consider a horn antenna which offers high antenna gain and high transmission power output [19].
2.1.2. Massive Antenna Structure (MAS). In order to densely integrate numerous antennas in BS and RBS, antenna miniaturization is necessary for design and implementation of the massive antenna structure. Due to the very short wavelength of the millimeter-wave band, the physical transceiver antenna sizes for BS and RBS can be greatly reduced. Based on the subminiature antennas, massive numbers of transceivers are densely integrated in a limited space of BS and RBS.

The MAS enables to support effective SDMA in combination with beamforming technology. Figure 2 shows the concept of SDMA in a BS adapting beamforming and massive antenna structure. In the conventional mobile communication networks, radio resources are used by being orthogonally divided in the whole coverage of BS (Figure 2(a)). On the other hand, if beamforming and MAS are adopted in the central network, BS operates a lot of antennas for each one to serve isolated region (Figure 2(b)). In this case, BS can spatially reuse its radio resources according to the region where a mobile device is located. As a result, SDMA with MAS can improve the spectral efficiency of the central network compared with that of the conventional $4 \mathrm{G}$ mobile communication networks.

2.1.3. Multilevel Relay. The millimeter-wave signal is difficult to be propagated toward the NLoS regions compared with the signal in the lower frequency band used in current $4 \mathrm{G}$ mobile communication systems $[14,15]$. In particular, the millimeterwave signal does not penetrate most solid materials and has weak diffraction at material surfaces. Especially in the urban environment, the propagated communication signals can be easily blocked by many skyscrapers which yield to create many coverage holes, where there will be no network coverage. Therefore, it is important to provide communication services to the coverage holes and in order to do so RBSs should be deployed under the coverage of the BS. Since many RBSs will increase the network deployment cost, the deployed location of the RBS should be carefully determined.

In complicated terrains such as downtown and apartment complexes, a one-hop relay is difficult to achieve LoS communication paths in the entire area. Therefore, as shown in Figure 3, the central network considers that RBSs 


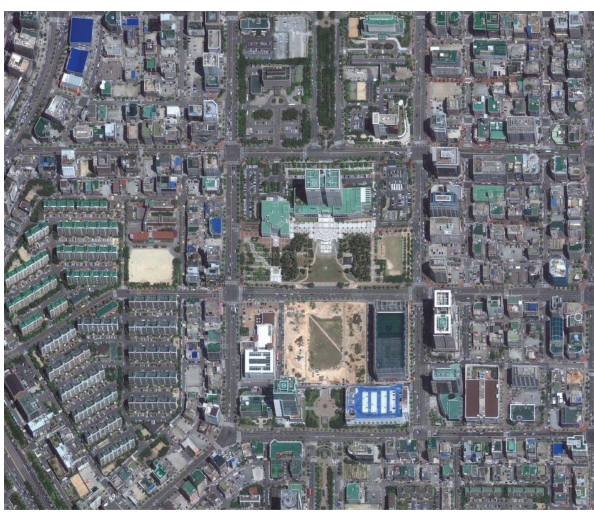

(a) Real map (Daejeon, Republic of Korea) (this Map is captured by using Google Maps service)

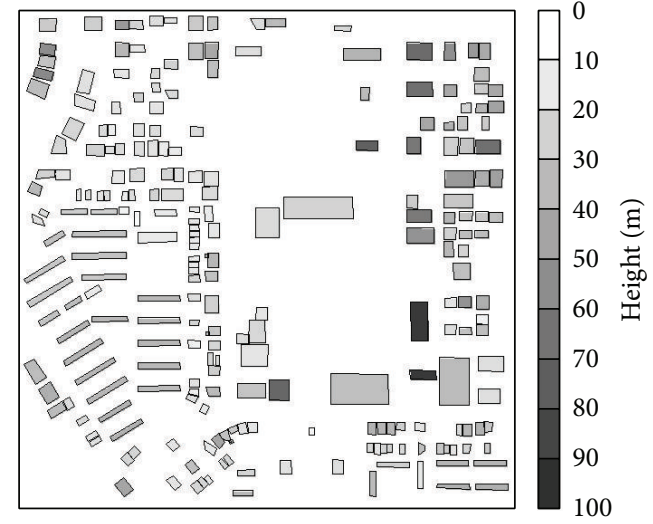

(b) 3D geographical environment

FIGURE 4: Region for performance evaluation.

are deployed with multiple level topologies to serve mobile devices located at coverage holes. RBSs are connected to BS/RBS at a higher level through wireless backhaul. The wireless backhaul supports flexible installation of RBSs at diverse locations such as towers, lamp posts, utility poles, and sides of buildings.

\section{Systematic Performance Evaluation}

3.1. Construction of the Simulation Environment. The terrain environment is a key factor affecting systematic performance of the central network because of the poor penetration, refraction, and diffraction of the millimeter-wave signal. In order to evaluate the realistic performance of the central network in real-world environments, we develop a systemlevel simulator by using $\mathrm{C}++$ language. Having graphical user interface (GUI) based on Microsoft Foundation Class (MFC), the system-level simulator is designed for performance evaluation of the central network as various deployment scenarios which user wants to analyze.

For the system-level simulator, we constructed a threedimensional (3D) geographical environment based on real geospatial information in a $1 \mathrm{~km} \times 1 \mathrm{~km}$ area at Daejeon City, Republic of Korea, as shown in Figure 4. The geospatial information was collected from actual measurement by the ETRI. Figure 4(b) shows that the 3D geographical environment includes the coordinates of buildings and their heights from the ground, where the height of each building from the ground is expressed as the depth of shade. In order to reduce the complexity of the simulation, we assume that the ground is flat and simplify the intricately shaped buildings into similar-sized square pillars.

In the central network, $1 \mathrm{GHz}$ downlink bandwidth in $27 \mathrm{GHz}$ frequency band is considered. For path-loss modeling in the wireless channel around $27 \mathrm{GHz}$, we use the wireless channel model of local multipoint distribution service (LMDS) which operates on $27.4 \mathrm{GHz}$ carrier frequency [20]. Since this LMDS channel model is based on enough measurements and the measured data is gathered in actual urban, suburban, and rural fields of Singapore, we expect that this model has sufficient accuracy for the wireless channel of the central network.In the LMDS model, received signal power $\left(P_{r}\right)$ according to the distance from the $\mathrm{BS}$ is calculated as follows:

$$
P_{r}=\mathrm{EIRP}+G_{r}-32.44-20 \log \left(f_{\mathrm{GHz}} \cdot d_{\mathrm{m}}\right)-L_{\mathrm{env}} .
$$

In here, effective isotropic radiated power (EIRP) is the effective transmission power radiated at the transmitter considering transmission power antenna gain, and $G_{r}$ is reception power antenna gain for received signal. $f_{\mathrm{GHz}}$ and $d_{\mathrm{m}}$ are carrier frequency $(\mathrm{GHz})$ and distance from the $\mathrm{BS}(\mathrm{m})$, respectively. $L_{\mathrm{env}}$ is the environmental loss of the propagated signal power according to environmental factors such as humidity and weather condition, which we have set to $6 \mathrm{~dB}$ here.

We use a ray-tracing method for modeling the wireless channel on the millimeter-wave band. In the millimeter-wave channel model, the LoS signal is considered as primary signal component. This is because the multiorder reflection signal experiences huge attenuation over $30 \sim 40 \mathrm{~dB}$ [21]. Furthermore, the millimeter-wave signal diffracted with incidence angle larger than $5^{\circ}$ is attenuated over $25 \mathrm{~dB}$ regardless of the surface materials of buildings [22]. However, a recent work shows that the first reflection signal is regarded as a reasonable component to create a communication link according to the material of the reflecting surface [14]. Therefore, based on that work, we include the first reflection signal in the channel model. For modeling the first reflected signal, we assume an ideal reflection case that the incident angle of signal is identical to that of the reflected signal. The first reflected signal is assumed to be additionally attenuated by $20 \mathrm{~dB}$ when the incident signal collides with a specular surface. By combining signal components and channel noise, signal-to-interference plus noise ratio (SINR) in the millimeter-wave channel model is calculated. In the model, the strongest component among all LoS and first reflection ones are regarded as the intended signal and the others as interferences. 


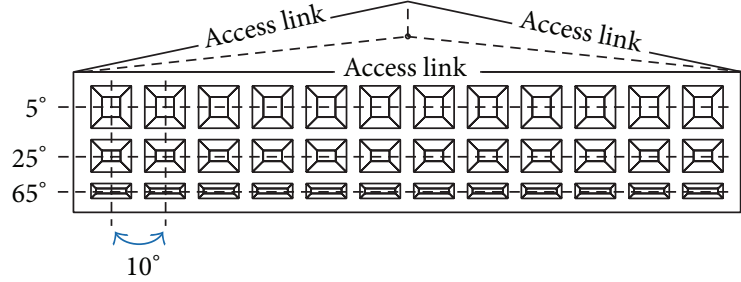

(a) Type A for BS

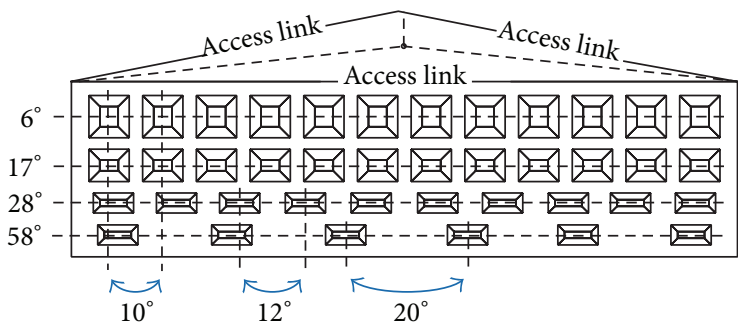

(c) Type E-1 for BS

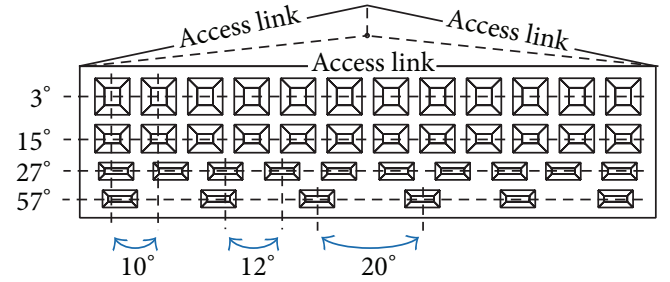

(b) Type D for BS

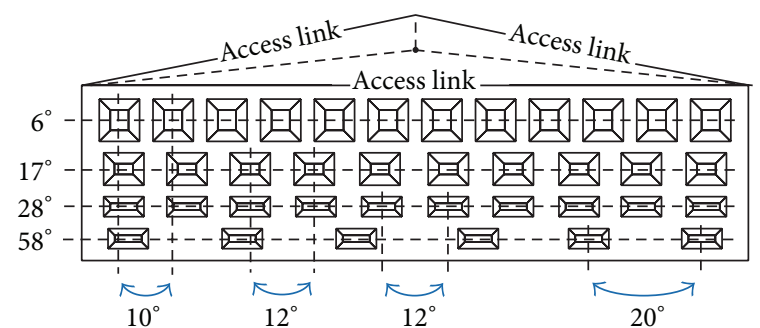

(d) Type E-2 for BS

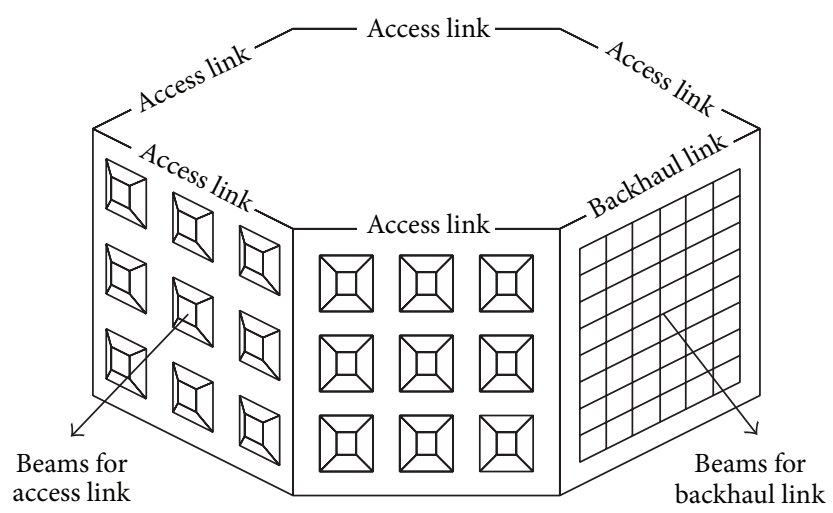

(e) Type B for RBS

FIGURE 5: Massive antenna structures for central network.

In order to obtain effective SDMA gain, we consider BS and RBS adopting MAS. Four types of MAS designs (types A, D, E-1, and E-2) and one type of MAS design (Type B) designed by ETRI are considered for BS and RBS, respectively. MASs for BS consist of three $120^{\circ}$ azimuth sectors and around 35 antennas are located with 3 or 4 rows at each sector side. On the other hand, MAS for RBS consists of six $60^{\circ}$ azimuth sectors, in which 5 sectors are used for access link communication and one sector is used for backhaul link communication. Nine antennas deployed by 3 by 3 matrix formation are located on the sector for access link communication. Each MAS has its individual configuration for tilting angle of deployed antennas. The tilting angle of each antenna is configured by considering interferences between neighboring antennas. In order to guarantee $18 \mathrm{~dB}$ SINR at the center point of each antenna, azimuth and elevation angles of each antenna are experimentally determined through the simulation. The structures and configurations of MAS types are in Figure 5 and in Table 1, respectively. In the simulation, BS and RBS are considered to radiate the signal with the antenna structure as shown in Figure 6. The radiation patterns are shaped by the transmission antenna gain, which depends on the angle of the transmitted signal. For the receiving antennas of devices, the receiving antenna gain is applied according to the direction of the incoming signal.

For performance evaluation of the central network, we use the random-walk model with simple modification [23]. In the mobility model, users only move outside the buildings, and the user entering the building changes its direction to outside the building. In addition, RSS-based beam selection is considered in the simulation. For this, we assume that mobile devices select the beam and BS/RBS to access the central network based on the RSS value. Mobile devices measure RSS of all available beams of BSs and RBSs, every beam selection period. If RSS value of the current beam is less than those of other beams, the mobile device will switch its access point to the beam giving the largest RSS (Handover). The other simulation parameters are in Table 2.

3.2. System Coverage of the Central Network. In order to achieve high performance of the central network operating at the millimeter-wave band, it is important to design the MAS. This is because of that the beam patterns according to the 


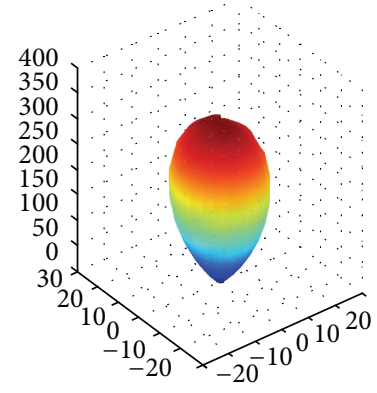

(a) Pattern A-1

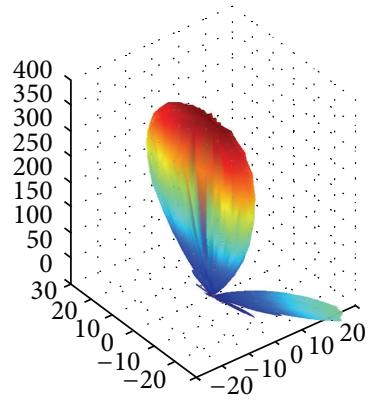

(e) Pattern B-2

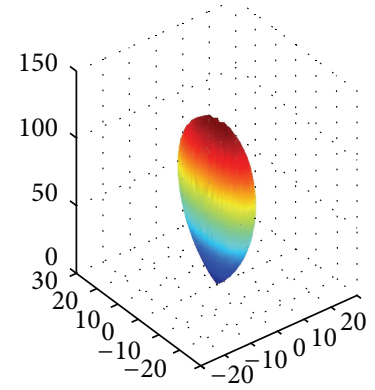

(b) Pattern A-2

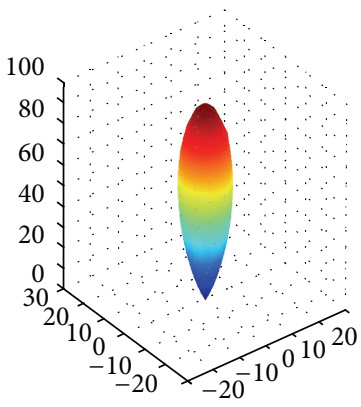

(f) Pattern C-1

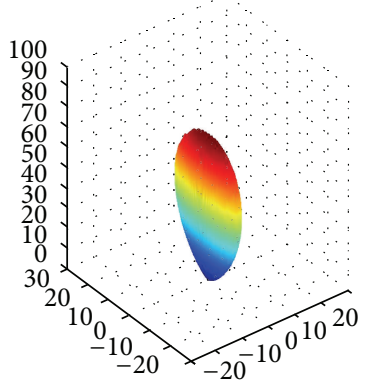

(c) Pattern A-3

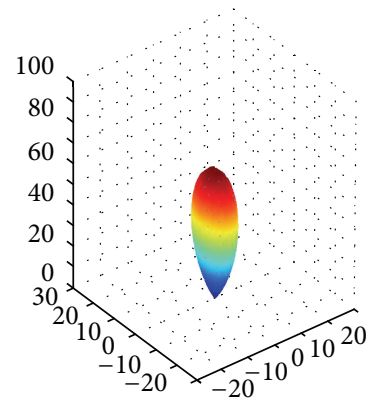

(g) Pattern C-2

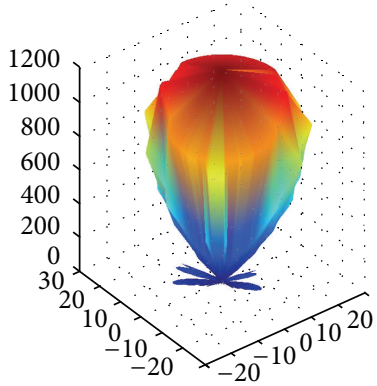

(d) Pattern B-1

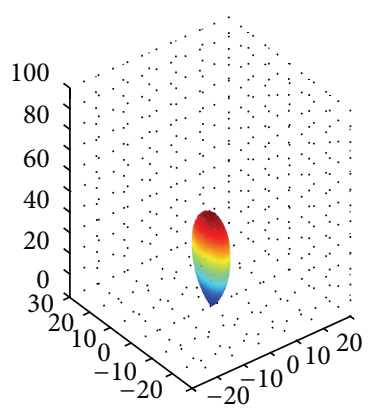

(h) Pattern C-3

Figure 6: Antenna gain response.

MAS closely correlate with the SINR level the mobile device experiences. The SINR level determines system coverage of the network. In the central network, the system coverage is defined as the region where the SINR level is expected to over $-0.8 \mathrm{~dB}$ which is minimally required level to decode wireless signal by considering modulation and coding schemes. Figure 7 and Table 3 show the system coverage and SINR patterns according to the MAS types. In the figure, regions covered by LoS communication paths and firstly reflected paths are represented by red and blue colors, and SINR level is represented by varying color grid. The BS using MAS Type A achieves the largest system coverage $(40.69 \%)$ in the simulation environment because its antennas radiate signal with wide-width (Patterns A-1, A-2, and A-3). In the same time, due to the wide-width radiation, severe interference is caused between neighboring beams and SINR level is the worst. On the other hand, since the other types of the MAS use narrow-width beam (patterns B-1 and B-2) compared with Type A, the SINR level is improved by mitigating the inter-beam interference. In addition, as the configuration of the MAS is adjusted, coverage holes occurring at the beam boundary regions can be reduced. For example, BS with Type E-2 can achieve considerable system coverage similar to that of Type A (39.93\%).

Despite adaptation of the well-designed the MAS, there are many coverage holes in the central network due to poor propagations of the millimeter-wave band. The coverage holes that are not covered by the BS can be minimized by deploying additional RBSs. For the RBS deployment, it is important for RBS to be located at a position enabling to obtain a clear and open view to the coverage holes. Figure 8 presents examples of system coverage expansions as varying with the number of RBSs. In the figure, more RBSs are deployed on the simulation area; both LoS and first reflection regions can broaden. For example, the BS with Type E-2 can achieve $86 \%$ system coverage with deployment of 10 RBSs. Especially, since the deployment of RBSs can broaden LoS region which can achieve good channel condition, it can be an effective solution for improving overall channel quality in the system coverage.

3.3. SINR of the Central Network. Figure 9 presents cumulative distribution function (CDF) of SINR measured by mobile devices according to their location. In the central network with the BS adopting MAS type A, the expected SINR of the mobile device is maximum $5 \mathrm{~dB}$ at the most as shown in Figure 9(a). On the other hand, in cases of types D, E-1, and E-2, the SINR level experienced by mobile devices increases up to $15 \mathrm{~dB}$. This is because inter-beam interferences are mitigated through the adjustment of the antenna configuration and tilting angle in types D, E-1, and E-2. Especially, types E-1 and E-2 are designed by focusing on minimizing interference occurring between antennas on the 3rd and 4th rows because the antennas mainly cover the central region of the cell where many mobile devices perform communications with very high-speed. When Type E-2 achieving the best performance is considered in the central network, poor SINR level on the system coverage can be overcome by deploying the additional RBS. As shown in 


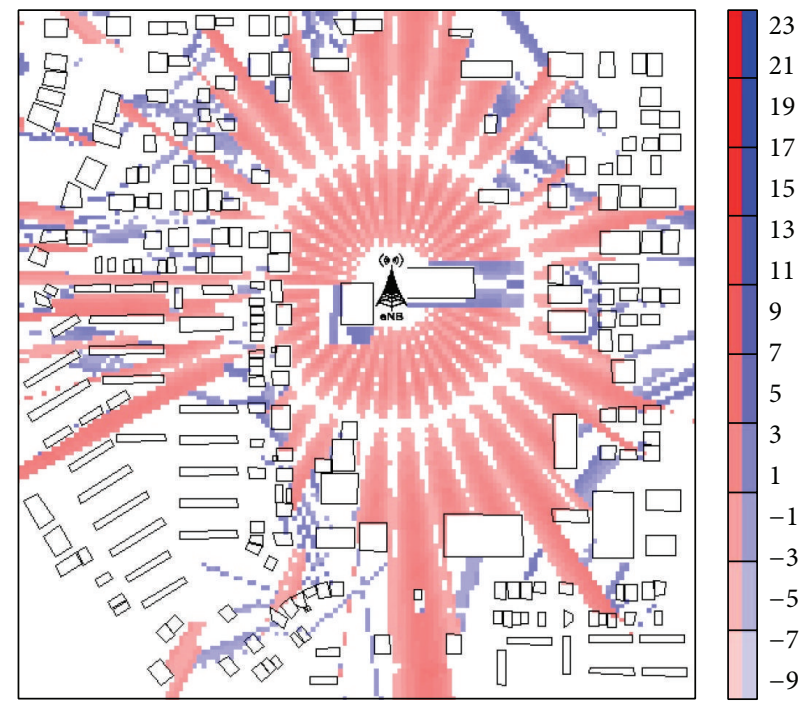

(a) Type A for BS (coverage: $40.69 \%$ )

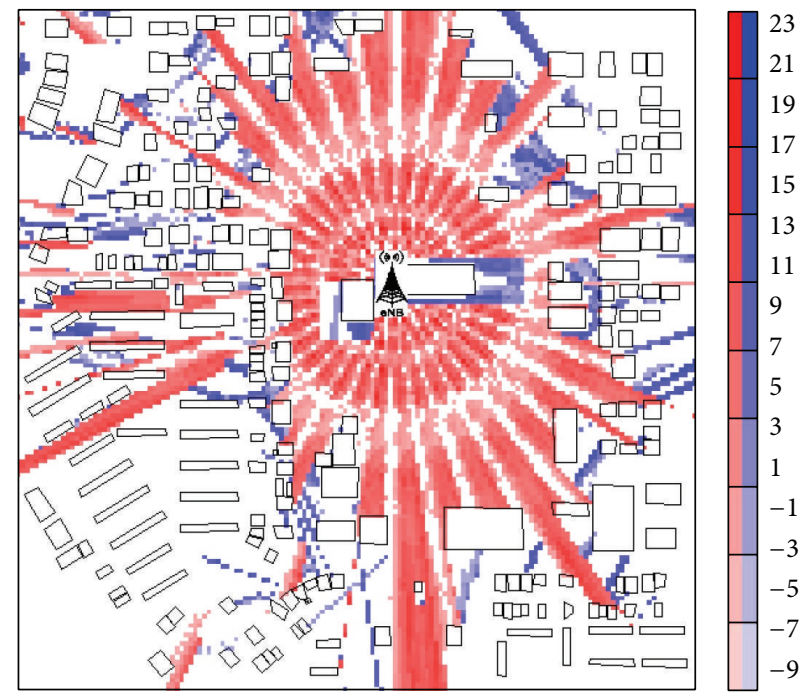

(c) Type E-1 for BS (coverage: $39.08 \%$ )

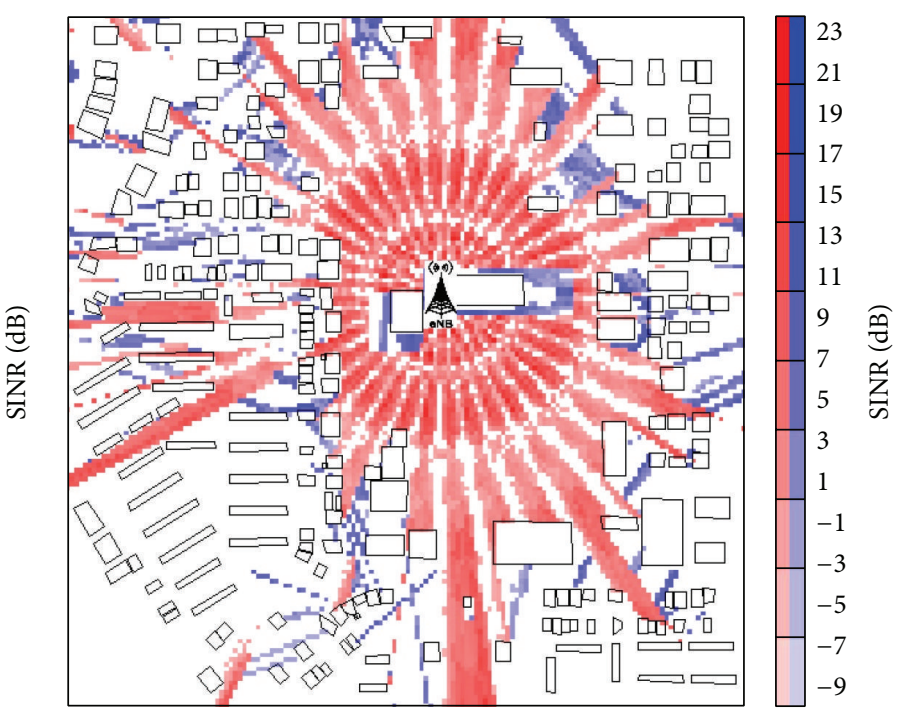

(b) Type D for BS (coverage: 37.02\%)

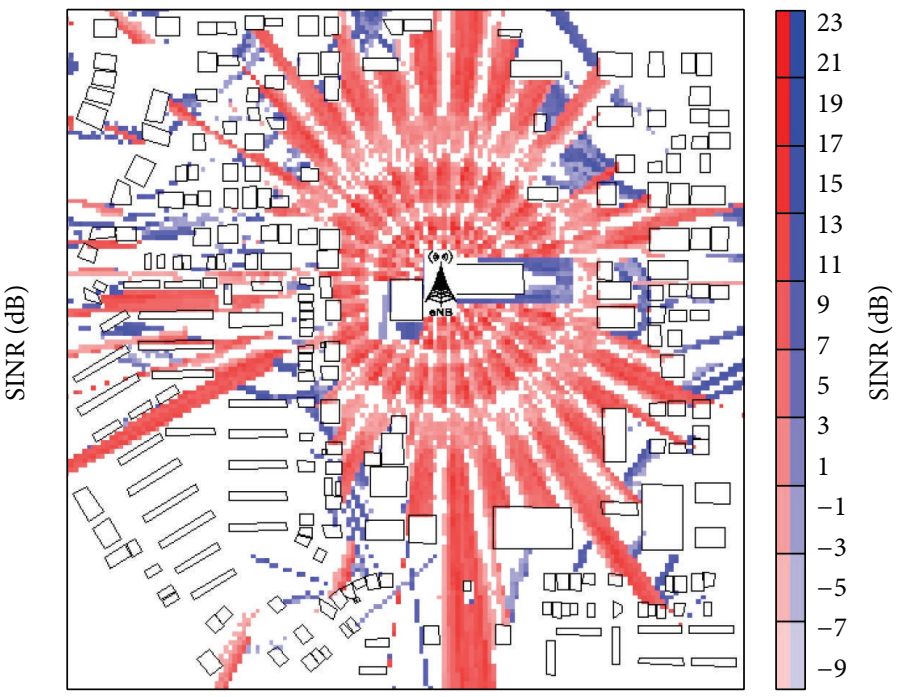

(d) Type E-2 for BS (coverage: 39.93\%)

FIGURE 7: System coverage and SINR patterns according to the MAS types.

Figure 9(b), the low SINR on the coverage hole is improved as the RBSs are additionally deployed.

3.4. System Capacity of the Central Network. It is important to verify how much mobile data traffic is accommodated in the central network. We define the system capacity as the total sum of mobile devices' downlink data rates when the available radio resources of the BS are fully utilized. So, in order to construct the above situations, we assume that buffers of the BS are always bursting with the data traffic to be transmitted to the mobile devices in the system coverage. The downlink data rate of mobile device $i$ provided from BS or RBS $k\left(C_{i, k}\right)$ is calculated as follows:

$$
C_{i, k}=B_{i, k} * \operatorname{SE}\left(\operatorname{SINR}_{i, k}\right) .
$$

In here, $B_{i}$ is the volume of radio resources which the BS or RBS $k$ allocates to device $i$, and $\operatorname{SINR}_{i, k}$ is achievable SINR of device $i$ from the BS or RBS $k$. SE $(*)$ is the spectral efficiency mapping function according to SINR level. Through the linklevel simulation performed by ETRI, optimal modulation and coding schemes (MCS) for SINR levels are found by considering bit error rate (BER) at the fading channel scenarios. Based on the MCS results, $\operatorname{SE}(*)$ is obtained as shown in Table 4.

The MAS is a key factor for achieving the capacity of the central network because the MAS directly affect transmitted signal power obtained by antenna gain and interference power occurring between neighboring beams. As shown in Figure 10, when the MAS type A using $10^{\circ}$-width beams is applied to the BS, the downlink system capacity is the lowest due to the inter-beam interference. On the other hand, the other types using $5^{\circ}$-width beams can improve system 
TABLE 1: Configurations of massive antenna structures.

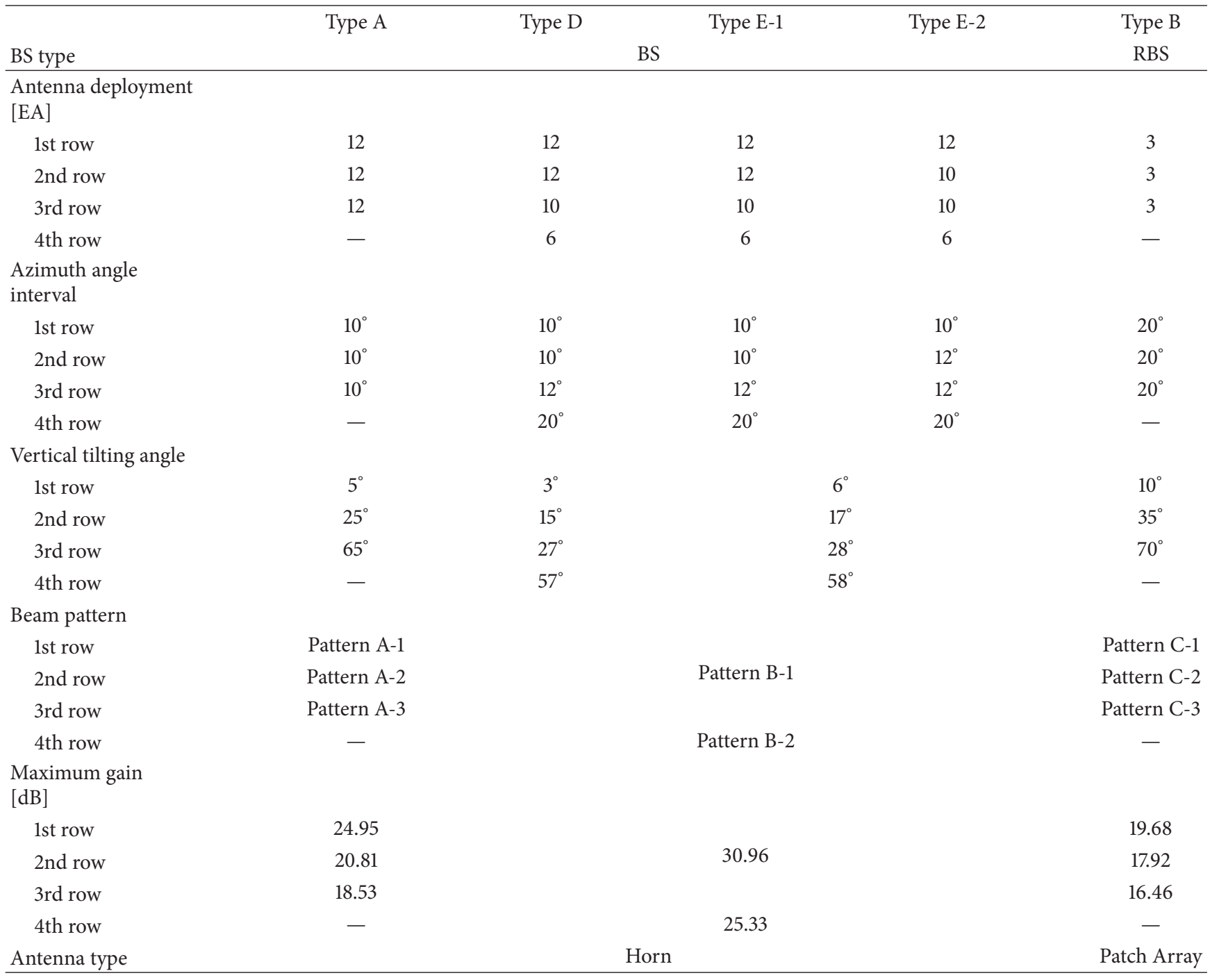

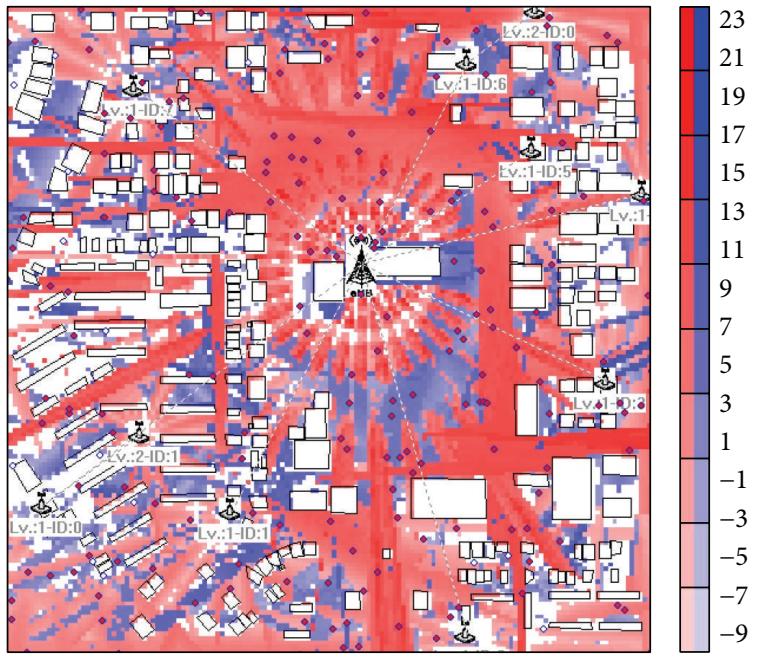

FIGURE 8: Coverage expansion of BS with 10 RBSs.
TABLE 2: Simulation parameters for the central network.

\begin{tabular}{lc}
\hline Parameter & Value \\
\hline MAS type for BS & Type A, Type D, Type E-1, Type E-2 \\
MAS type for RBS & Type B \\
BS height & $30[\mathrm{~m}]$ \\
RBS height & $10[\mathrm{~m}]$ \\
BS transmission power & $30[\mathrm{dBm}]$ \\
RBS transmission power & $30[\mathrm{dBm}]$ \\
Carrier frequency & $27[\mathrm{GHz}]$ \\
Downlink bandwidth & $1[\mathrm{GHz}]$ \\
Channel environmental loss & $6[\mathrm{~dB}]$ \\
Reflection attenuation & $20[\mathrm{~dB}]$ \\
Beam selection period & $1[\mathrm{~ms}]$ \\
\hline
\end{tabular}

capacity two times more than that of Type A as the interbeam interference is mitigated. Especially, in case 100 mobile 


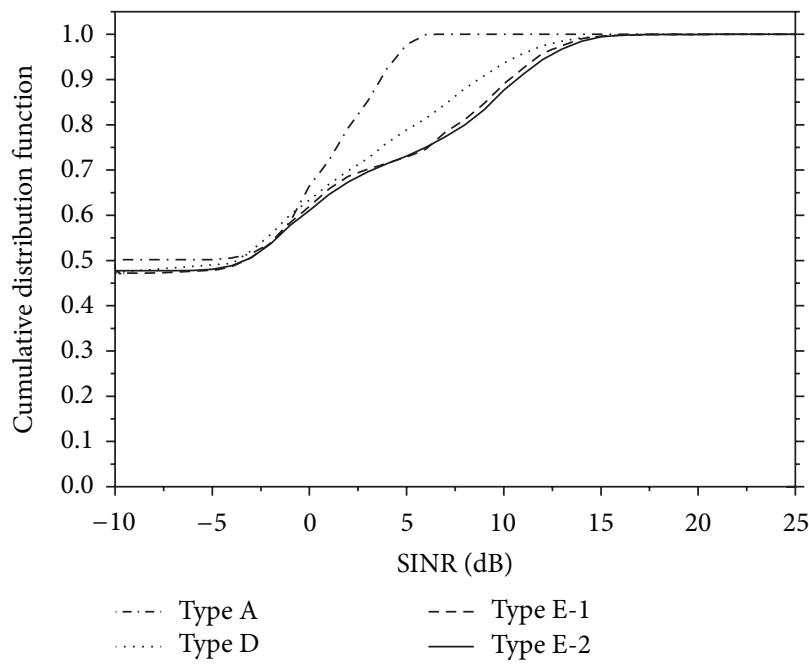

(a) SINR CDF according to MAS type

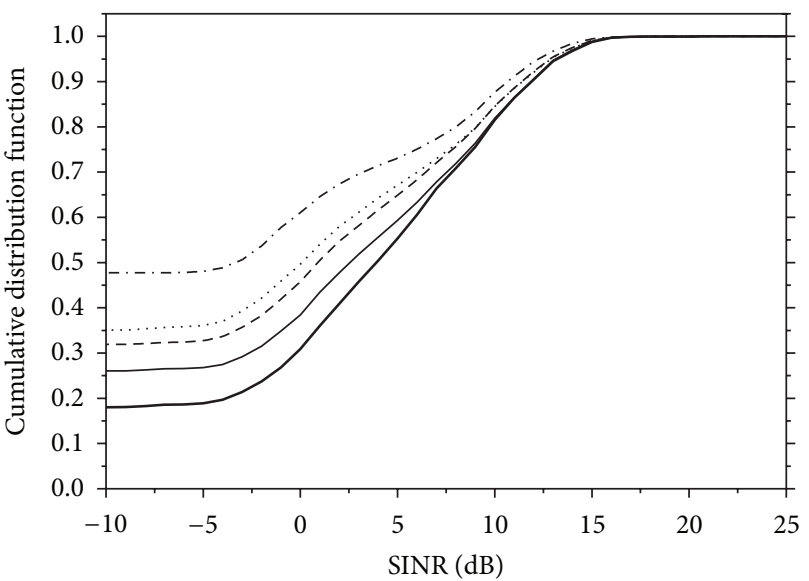

-... 1 BS with no RBS _ 1 BS with 3 RBSs

…. 1 BS with 1 RBS

- 1 BS with 4 RBSs

- 1 BS with 2 RBSs

(b) SINR CDF according to the number of RBSs

FIGURE 9: Cumulative distribution function of SINR measured by mobile devices.

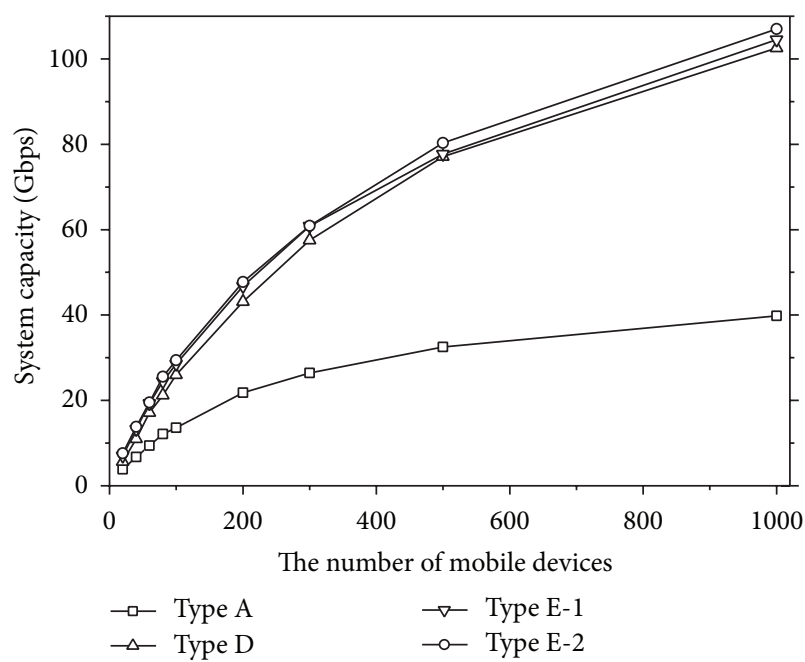

(a) Total system capacity

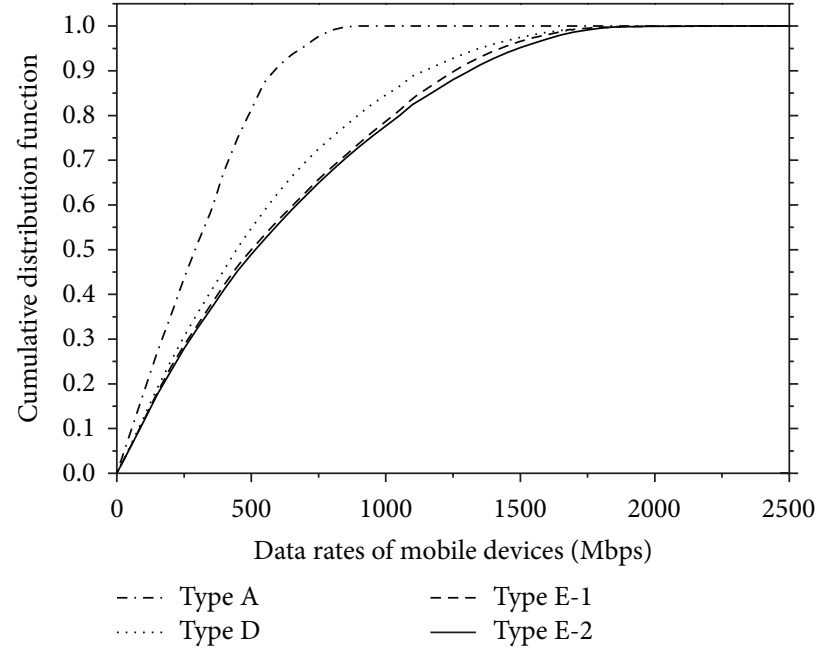

(b) $\mathrm{CDF}$ of data rate per mobile device

FIGURE 10: System capacity according to MAS types for BS.

devices are deployed, the system capacity of types E-1 and E-2 can be improved about 110 percent compared with that of Type A. This is because interferences occurring between beams on the $3 \mathrm{rd}$ and 4 th rows are mitigated in cases of types E-1 and E-2 due to rearrangement of antennas. As shown in Figure 10(b), peak downlink data rate of the served mobile device is improved from $900 \mathrm{Mbps}$ on Type A to $1.8 \mathrm{Gbps}$ on Type E-2.

Figure 11 shows the variation of the system capacity according to the number of RBSs. Since the MAS and beamforming are adopted in the central network, radio resources are spatially reused and the system capacity can increase as the number of mobile devices increases. However, deploying new RBSs in existing network can cause interference to the existing BS and RBSs. So in order to minimize this interference, the new RBSs should be deployed on locations that do not overlap the coverage area of existing BS and RBSs. Considering the inter-BS/RBS interference while deploying new RBS can improve the performance of the mobile devices on the coverage holes. Figure 11(b) represents CDF of data rates that each mobile device can achieve with varying number of RBSs. On the BS, the average number of served mobile devices increases as the additional RBS is installed. As a result, the usable volume of resources per mobile device can decrease and the ratio of mobile devices served with low data rates can be slightly reduced. However, since the RBS can 


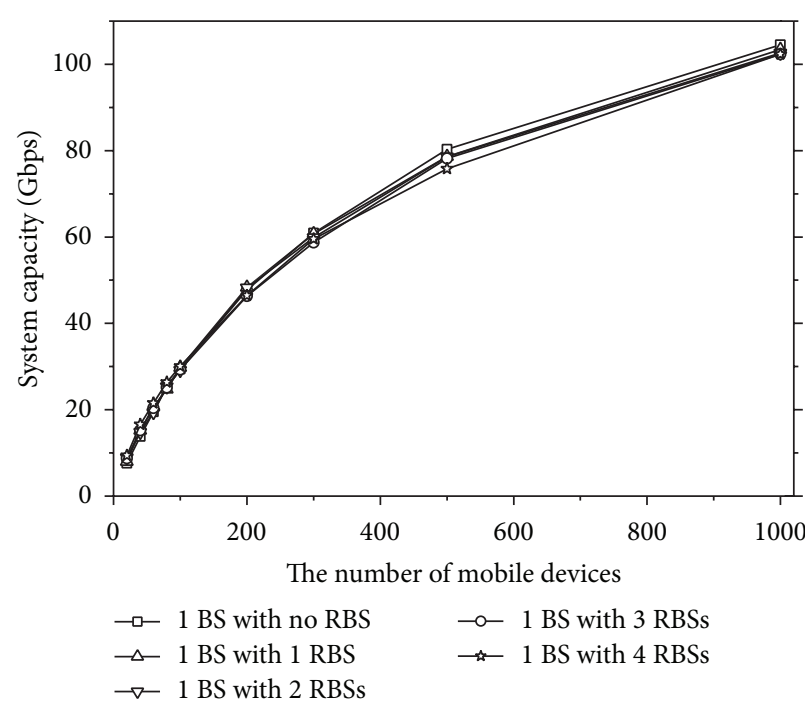

(a) Total system capacity

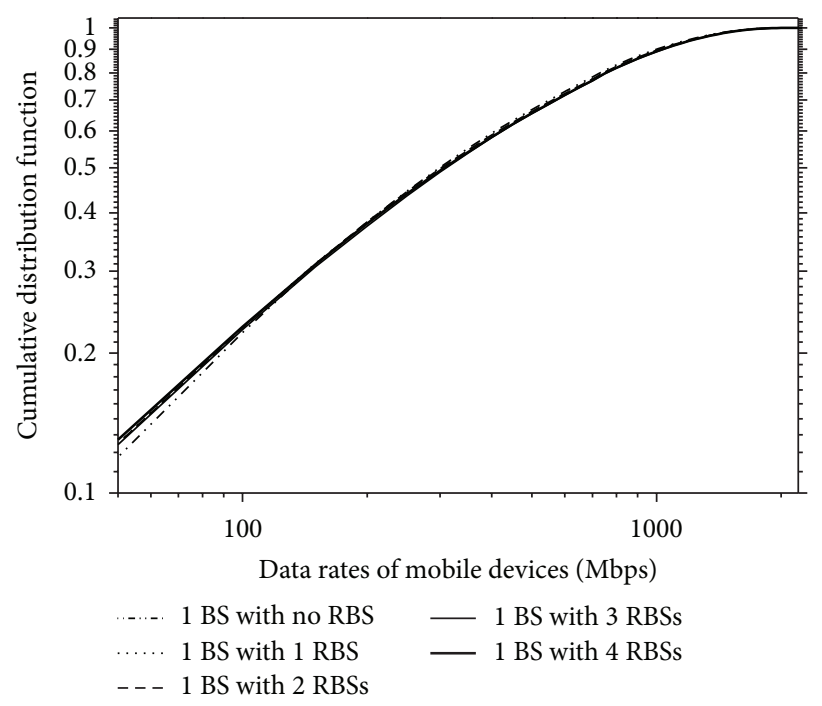

(b) $\mathrm{CDF}$ of data rate per mobile device

FIGURE 11: System capacity according to the number of RBSs.

TABLE 3: System coverage according to the MAS types.

\begin{tabular}{lccc}
\hline MAS type & $\begin{array}{c}\text { LoS region ratio } \\
{[\%]}\end{array}$ & $\begin{array}{c}\text { First reflection region ratio } \\
{[\%]}\end{array}$ & $\begin{array}{c}\text { Total system coverage } \\
{[\%]}\end{array}$ \\
\hline A & 32.74 & 7.94 & 40.69 \\
D & 29.90 & 7.13 & 37.02 \\
E-1 & 31.57 & 7.50 & 39.08 \\
E-2 & 32.43 & 7.50 & 39.93 \\
\hline
\end{tabular}

TABLE 4: Reference MCS-SINR table at fading channel with real channel estimation.

\begin{tabular}{|c|c|c|c|c|c|c|c|c|c|c|c|c|c|c|c|c|c|c|}
\hline \multirow{2}{*}{$\begin{array}{l}\text { Modulation } \\
\text { Code rate }\end{array}$} & \multicolumn{6}{|c|}{ QPSK } & \multicolumn{6}{|c|}{ 16QAM } & \multicolumn{6}{|c|}{ 64QAM } \\
\hline & $1 / 3$ & $1 / 2$ & $2 / / 3$ & $3 / 4$ & $4 / 5$ & $5 / 6$ & $1 / 3$ & $1 / 2$ & $2 / / 3$ & $3 / 4$ & $4 / 5$ & $5 / 6$ & $1 / 3$ & $1 / 2$ & $2 / / 3$ & $3 / 4$ & $4 / 5$ & $5 / 6$ \\
\hline $\begin{array}{l}\text { SINR } \\
{[\mathrm{dB}]}\end{array}$ & -0.8 & 1.7 & 3.7 & 4.7 & 5.5 & 6.0 & 4.2 & 7.2 & 9.9 & 11.2 & 12.1 & 12.7 & 8.3 & 12.0 & 15.6 & 17.2 & 18.9 & 20.0 \\
\hline $\begin{array}{l}\text { Spectral efficiency } \\
\text { [bps/HZ] }\end{array}$ & 0.41 & 0.62 & 0.82 & 0.93 & 0.99 & 1.03 & 0.81 & 1.24 & 1.65 & 1.86 & 1.98 & 2.07 & 1.24 & 1.85 & 2.46 & 2.76 & 2.97 & 3.10 \\
\hline
\end{tabular}

improve SINR level of the mobile device especially located at cell-boundary region. This means that the data rate of mobile devices located at the cell-boundary region can be improved.

\section{Conclusions}

The millimeter-wave band in the $30-300 \mathrm{GHz}$ range is a primary candidate for a new radio band that can significantly improve the capacity of the central network for 5G mobile communications. However, due to the poor propagation characteristics of millimeter waves, the central network considered MAS with beamforming enabling to obtain transmitting and receiving gain. In this paper, through the system-level simulation, we evaluated the system coverage and capacity of the central network according to the configuration of MAS. Simulation results showed that the antenna configurations such as arrangement, tilting angle, and spacing are tightly coupled with the coverage and capacity of the system. In general, the antenna radiating widewidth beam can broaden system coverage, while the narrowwidth beam enables to improve channel quality and system capacity by mitigating interbeam interference. Therefore, for high-performance $5 \mathrm{G}$ mobile communication system, it is important to find optimal beam pattern and configuration for the antenna structure consisting of base stations and relay base stations. We hope that our research can be a useful contribution for designing $5 \mathrm{G}$ system architecture and antenna structure.

\section{Conflict of Interests}

The authors declare that there is no conflict of interests regarding the publication of this paper. 


\section{Acknowledgment}

This research was funded by the Ministry of Science, ICT \& Future Planning (MSIP), Korea, in the ICT R\&D Program 2014.

\section{References}

[1] "Cisco Visual Networking Index: Global Mobile Data Traffic Forecast Update," 2010.

[2] Qualcomm Incorporated, "The 1000x Data Challenge: More Small Cells, More Spectrum, Higher Efficiency," 2013.

[3] D. Astély, E. Dahlman, A. Furuskär, Y. Jading, M. Lindström, and S. Parkvall, "LTE: the evolution of mobile broadband," IEEE Communications Magazine, vol. 47, no. 4, pp. 44-51, 2009.

[4] S. Parkvall, A. Furuskär, and E. Dahlman, "Evolution of LTE toward IMT-advanced," IEEE Communications Magazine, vol. 49, no. 2, pp. 84-91, 2011.

[5] F. Khan, Z. Pi, and S. Rajagopal, "Millimeter-wave mobile broadband with large scale spatial processing for $5 \mathrm{G}$ mobile communication," in Proceedings of the 50th Annual Allerton Conference on Communication, Control, and Computing, Allerton, pp. 1517-1523, October 2012.

[6] F. Khan and Z. Pi, "mmWave mobile broadband (MMB): unleashing the 3-300 GHz spectrum," in Proceedings of the 34th IEEE Sarnoff Symposium, pp. 1-6, Princeton, NJ, USA, May 2011.

[7] K. I. Pedersen, P. E. Mogensen, and J. Ramiro-Moreno, "Application and performance of downlink beamforming techniques in UMTS," IEEE Communications Magazine, vol. 41, no. 10, pp. 134-143, 2003.

[8] L. Liu, R. Chen, S. Geirhofer, K. Sayana, Z. Shi, and Y. Zhou, "Downlink MIMO in LTE-advanced: SU-MIMO versus MUMIMO," IEEE Communications Magazine, vol. 50, no. 2, pp. 140-147, 2012.

[9] S. D. Blostein and H. Leib, "Multiple antenna systems: Their role and impact in future wireless access," IEEE Communications Magazine, vol. 41, no. 7, pp. 94-101, 2003.

[10] C. Hoymann, W. Chen, J. Montojo, A. Golitschek, C. Koutsimanis, and X. Shen, "Relaying operation in 3GPP LTE: challenges and solutions," IEEE Communications Magazine, vol. 50, no. 2, pp. 156-162, 2012.

[11] Y. Yang, H. Hu, J. Xu, and G. Mao, "Relay technologies for WiMAX and LTE-advanced mobile systems," IEEE Communications Magazine, vol. 47, no. 10, pp. 100-105, 2009.

[12] "Propagation data and prediction methods for the planning of short-range outdoor radio communication systems and radio local area networks in the frequency range $300 \mathrm{MHz}$ to 100 GHz," ITU-R P.1411-6, 2012.

[13] I. Rubin, H. Chang, and R. Cohen, "Optimal relay deployment for multihop multicast scheduling in cellular wireless networks," in Proceedings of the IEEE Globecom Workshops (GC Wkshps '12), pp. 1166-1171, December 2012.

[14] J. N. Murdock, E. Ben-Dor, Y. Qiao, J. I. Tamir, and T. S. Rappaport, "A $38 \mathrm{GHz}$ cellular outage study for an urban outdoor campus environment," in Proceedings of the IEEE Wireless Communications and Networking Conference (WCNC '12), pp. 3085-3090, Shanghai, China, April 2012.

[15] Z. Pi and F. Khan, "An introduction to millimeter-wave mobile broadband systems," IEEE Communications Magazine, vol. 49, no. 6, pp. 101-107, 2011.
[16] R. Merritt, “5G Work Officially Begins in Europe”, 2013, http://www.eetimes.com/document.asp?doc_id=1320437.

[17] https://http://www.metis2020.com/.

[18] A. Osseiran, "The $5 \mathrm{G}$ mobile and wireless communications system," in Proceedings of the ETSI Summit on Future Mobile and Standards for 5G, November 2013.

[19] C. A. Balanis, Antenna Theory: Analysis and Design, Wiley, 3rd edition, 2005.

[20] P. Soma, L. C. Ong, S. Sun, and M. Y. W. Chia, "Propagation measurements and modeling of LMDS radio channel in Singapore," IEEE Transactions on Vehicular Technology, vol. 52, no. 3, pp. 595-606, 2003.

[21] A. Hayn, R. Bose, and R. Jakoby, "Multipath propagation and LOS interference studies for LMDS architecture," in Proceedings of the International Conference on Antennas and Propagation, vol. 2, pp. 686-690, April 2001.

[22] P. A. Tenerelli and C. W. Bostian, "Measurements of $28 \mathrm{GHz}$ diffraction loss by building corners," in Proceedings of the 9th IEEE International Symposium on Personal, Indoor and Mobile Radio Communications (PIMRC '98), vol. 3, pp. 1166-1169, Boston, Mass, USA, September 1998.

[23] T. Camp, J. Boleng, and V. Davies, "A survey of mobility models for ad hoc network research," Wireless Communications and Mobile Computing, vol. 2, no. 5, pp. 483-502, 2002. 

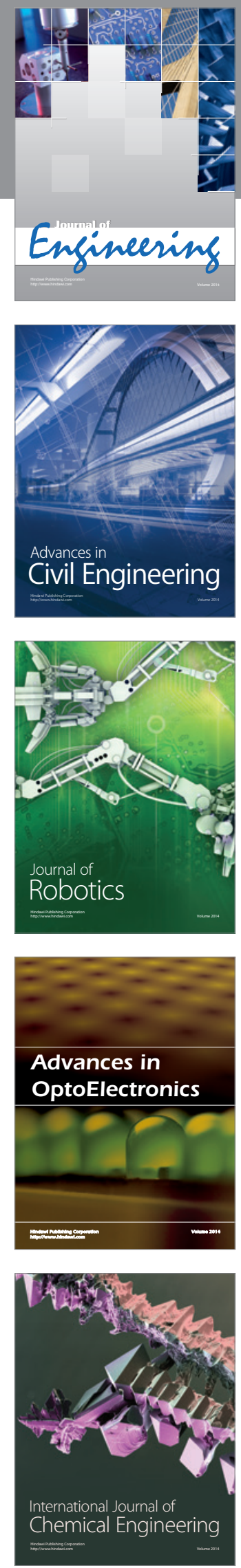

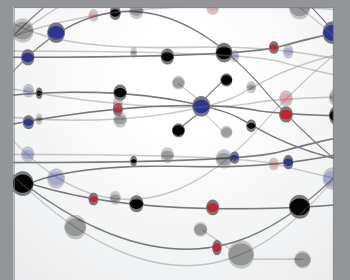

The Scientific World Journal
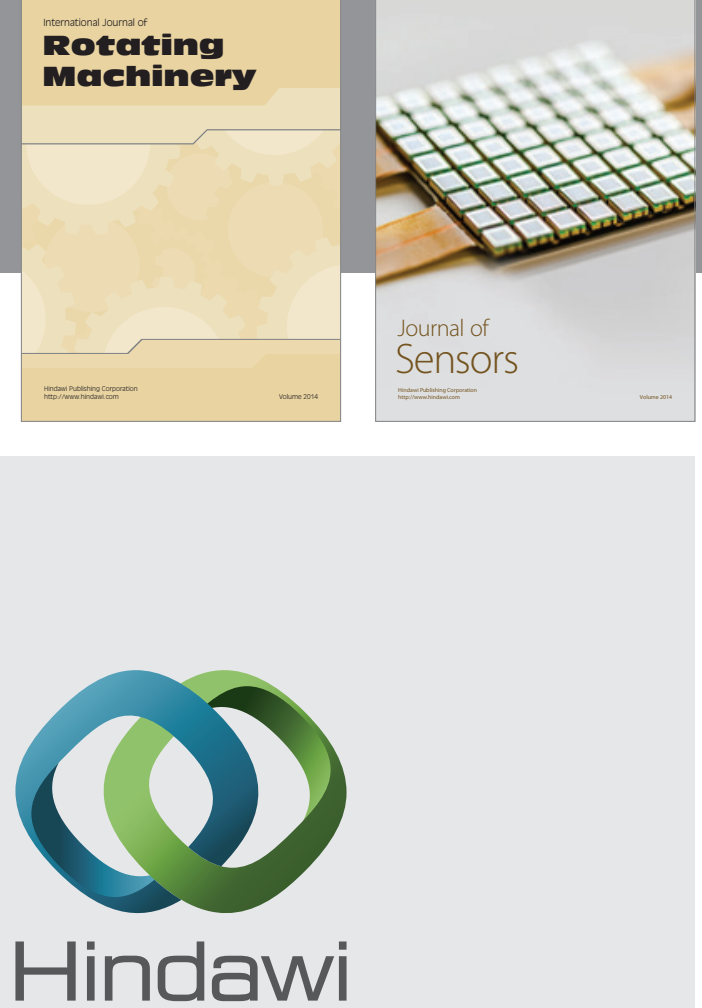

Submit your manuscripts at http://www.hindawi.com
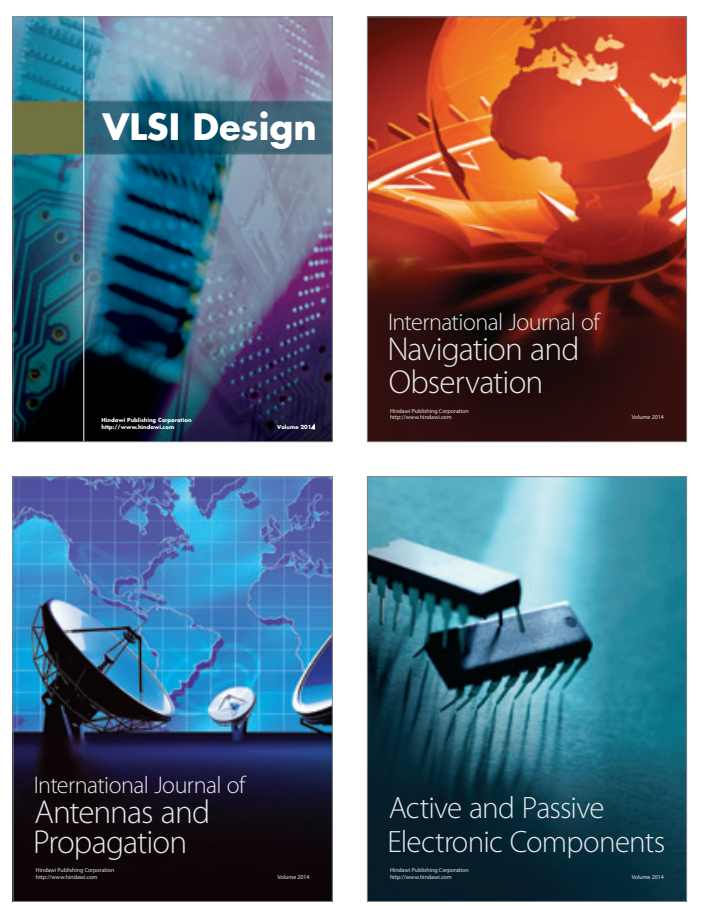
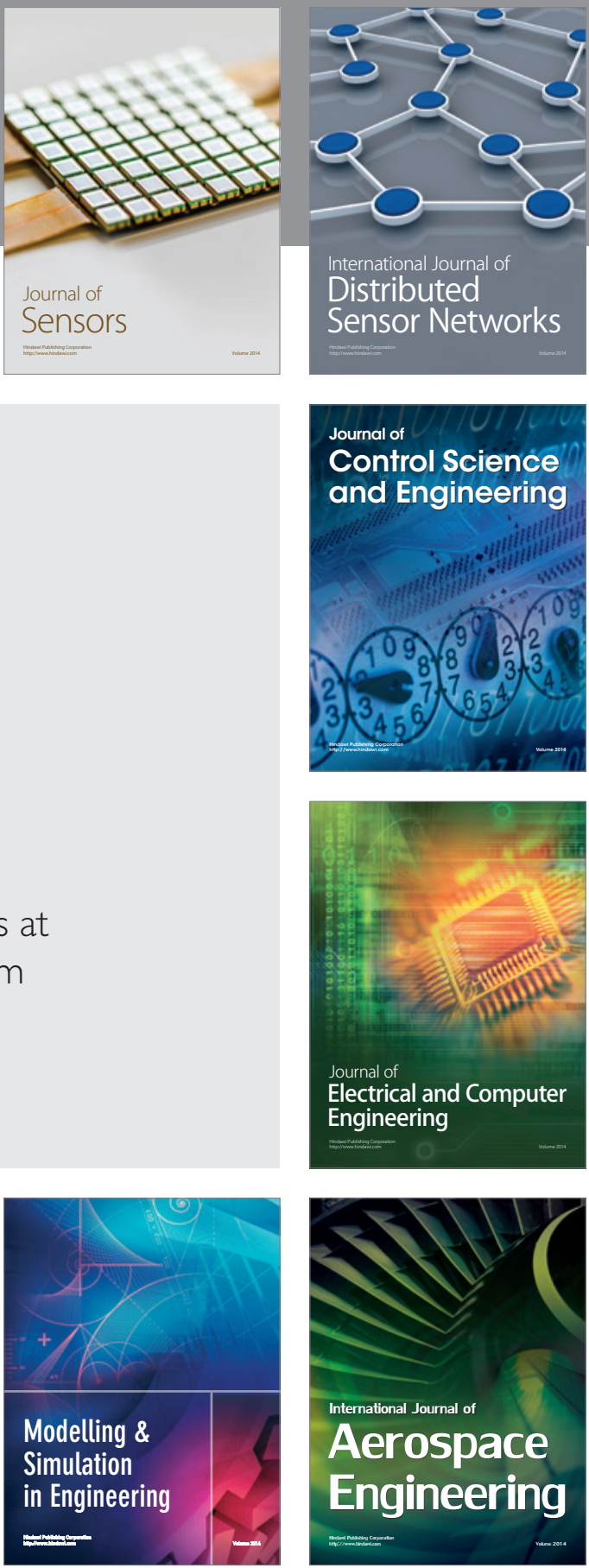

Journal of

Control Science

and Engineering
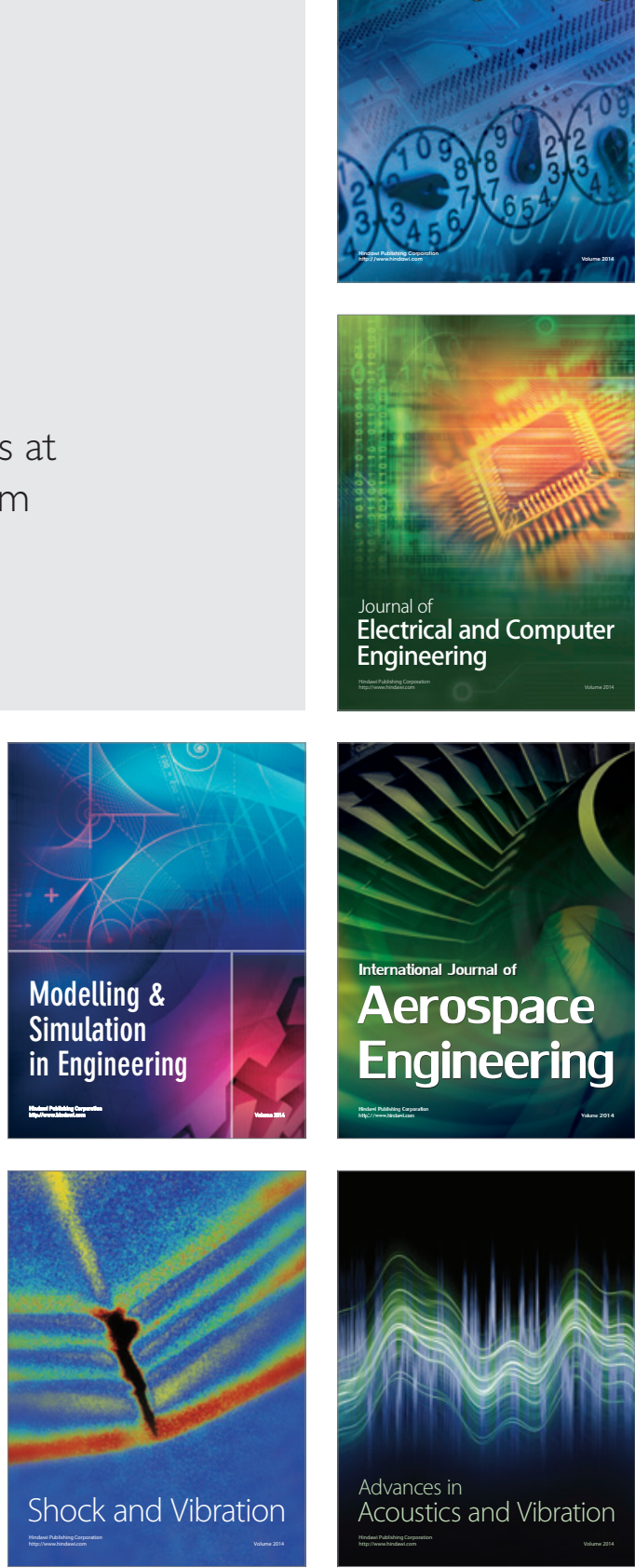